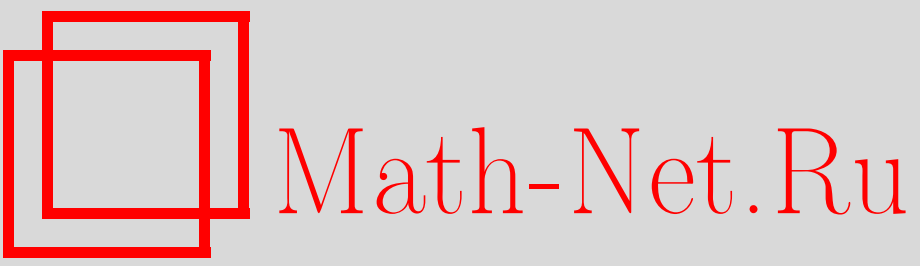

И. М. Кричевер, С. П. Новиков, Голоморфные расслоения и разностные скалярные операторы: одноточечные конструкции, УМH, 2000, том 55, выпуск 1, 187188

DOI: https://doi.org/10.4213/rm258

Использование Общероссийского математического портала Math-Net.Ru подразумевает, что вы прочитали и согласны с пользовательским соглашением http://www . mathnet.ru/rus/agreement

Параметры загрузки:

IP : 3.89 .185 .249

26 апреля 2023 г., 02:20:45 


\title{
ГОЛОМОРФНЫЕ РАССЛОЕНИЯ И РАЗНОСТНЫЕ СКАЛЯРНЫЕ ОПЕРАТОРЫ: ОДНОТОЧЕЧНЫЕ КОНСТРУКЦИИ
}

\author{
И. М. КРИчевЕР, С.П. Новиков
}

Рассмотрим аналогично [1] неособую алгебраическую кривую $Г$ с отмеченной точкой $P_{0}=\infty$ и локальной координатой $z=k^{-1}, z\left(P_{0}\right)=0$. Обозначим через $A=A\left(\Gamma, P_{0}\right)$ кольцо алгебраических функций с единственным полюсом в $P_{0}$. Зададим "данные обратной задачи" в виде набора точек $\left(\gamma_{1}, \ldots, \gamma_{l g}\right)$, где $l$ - это "ранг" и $g$ - род кривой $\Gamma$, параметров $\alpha_{s j}, s=1, \ldots, l g$, $j=1, \ldots, l-1$ и матричной $l \times l$-функции $\chi_{n}^{(0)}(k)(n \in \mathbb{Z})$ с ненулевыми элементами только $\chi^{(0) p, p+1}=1, p \leqslant l-1, \chi^{(0) l q}=a_{q}, q=1, \ldots, l$, где $a_{q}$ - это полиномы от $k$, зависящие от $n$.

ТеОРема 1. Для любого вектора $\eta_{0}$ и данных общего положения существует и единственна вектор-функция “Бейкера-Ахиезера" $\psi_{n}(P), P \in \Gamma$, мероморфная на $\Gamma \backslash P_{0}$, с полюсами первого порядка в точках $\gamma_{s}$, где вычеты связаны соотночениями $\left(\operatorname{res} \gamma_{s} \psi^{q+1}\right)=\alpha_{s q}\left(\operatorname{res} \gamma_{s} \psi^{1}\right), s=1, \ldots, l g, q=1, \ldots, l-1$. В окрестности точки $\infty=P_{0}$ вектор-функция $\psi$ имеет асимптотику $\psi=\left[\eta_{0}+\sum_{s \geqslant 1} \eta_{s n} k^{-s}\right] \Psi^{(0)}, \Psi_{x}^{(0)}=\chi^{(0)} \Psi^{(0)}$ или $\Psi_{n+1}^{(0)}=\chi_{n}^{(0)} \Psi_{n}^{(0)}, \Psi^{(0)}-$ это $l \times l$-матрица.

Теорема 2. Пусть матрица $\chi^{(0)}$ зависит от $k$ так, что лишь одна из функций $a_{j n}(k)$ имеет вид $a_{j}=k-v_{j, n+1}^{(0)}$, а все остальные $a_{q}$ не зависят от $k$ при $q \neq j$. Тогда для любой функции $f(P) \in A\left(\Gamma, P_{0}\right)$ с полюсом порядка $\tau$ найдется единственный оператор $L_{f}$ вида

$$
L_{f}=\sum_{-M}^{+N} u_{p n} T^{p},
$$

əде $N=\tau(l-j+1), M=\tau(j-1), T \psi_{n}=\psi_{n+1}, M+N=\tau l$, такой, что вектор-функция Бейкера-Ахиезера $\psi$, построенная в теореме 1 , где $\eta_{0}=\left(\eta_{0}^{q}\right), \eta_{0}^{q}=\delta^{q j}$, удовлетворяет уравнению

$$
L_{f} \psi=f \psi \text {. }
$$

ЗАмечание. Для $j=1$ это утверждение содержится в [1], [2] в непрерывном случае. Напомним, что $(\alpha, \gamma)$ - это “параметры Тюрина", характеризующие оснащенное голоморфное стабильное расслоение $\eta$, где $c_{1}(\operatorname{det} \eta)=l g$. Все известные ранее конструкции разностных коммутирующих операторов (ранга 1) требовали не менее двух "бесконечных" точек на кривой $\Gamma$; отметим, что симметричные операторы $M=N$ возможны лишь для четного ранга $l=2 j-2$.

Следуя идее [1], рассмотрим многопараметрическую вектор-функцию Бейкера-Ахиезера. Она задается теми же, что и в теореме 1 , данными $\left(\Gamma, P_{0}, \gamma_{s}, \alpha_{s j}, z=k^{-1}\right)$, но для каждой новой переменной $t_{p}$ задается дополнительно своя матрица $M^{(0 p)}, p=1,2, \ldots$ При этом “затравочная" матрица $\Psi_{n}^{(0)}$ определяется из уравнений $\left(t=\left(t_{1}, t_{2}, \ldots\right)\right)$ :

$$
\Psi_{n+1}^{(0)}=\chi_{n}^{(0)} \Psi_{n}^{(0)}, \quad \Psi_{t_{p}}^{(0)}=M^{(0 p)} \Psi^{(0)}, \quad p=1,2, \ldots
$$

где $\chi^{(0)}$ выбраны как в теореме 2.

ТЕОРема 3. Для любого $l \geqslant 2$ можно вибрать матрици $M^{(0 p)}, p=1,2, \ldots$, так, что вектор-функция Бейкера-Ахиезера $\psi$ определяют решения иерархий двумеризованной цепочки Тода при любъх данных обратной задачи $\left(\Gamma, P_{0}, z=k^{-1}, \gamma_{s}, \alpha_{s q}\right)$. (Решение, определяемое функцией $\psi$, мы назовем решением ранга l.) 
ПримеР. Пусть $g=1, l=2, a_{1}=-c_{n+1}^{(0)}, a_{2}=k-v_{n+1}^{(0)}$, заданы данные $\left(\gamma_{1}, \gamma_{2}, \alpha_{1}, \alpha_{2}\right)$ и функция $f(P)=\lambda=k^{2}$ на Г. Из вектора Бейкера-Ахиезера $\Psi_{n}$ сделаем матрицу $\widehat{\Psi}_{n}$ со строками $\psi_{n}, \psi_{n+1}$. Мы имеем $\widehat{\Psi}_{n+1}=\chi_{n} \widehat{\Psi}_{n}$, где $\chi_{n}=\left(-c_{n+1}^{0,1}, k-v_{n+1}\right)+O\left(k^{-1}\right)$. Полюсы $\chi_{n}$ лежат в точках $\gamma_{s n}$, где $\gamma_{s 0}=\gamma_{s}, s=1,2$. Нули $\left(\operatorname{det} \chi_{n}\right)$ лежат в точках $\gamma_{s, n+1}$. При этом $\alpha_{s n} \operatorname{res}_{s n} \chi^{i 1}=\operatorname{res} \gamma_{s n} \chi^{i 2}, i=1,2, \alpha_{s, n+1}=-\chi^{22}\left(\gamma_{s, n+1}\right)$. Величина $\gamma_{1 n}+\gamma_{2 n}=c$ не зависит от $n$. Операторы $L_{f}$ эффективно вычисляются. Для $f=\lambda=-P(z)$ и $c=0$ мы имеем симметризуемый оператор четвертого порядка

$$
\begin{gathered}
\lambda \psi_{n}=L_{\lambda} \psi_{n}=\left[\left(L_{2}\right)^{2}+u_{n}\right] \psi_{n}, \quad L_{2} \psi_{n}=\psi_{n+1}+v_{n} \psi_{n}+c_{n} \psi_{n-1}, \\
u_{n}=-\left[\wp\left(\gamma_{n-1}\right)+\wp\left(\gamma_{n-2}\right)\right]+b_{n-1}+b_{n-2}, \quad \wp(z)=-\zeta^{\prime}(z), \\
b_{n}=2 \wp^{\prime}\left(\gamma_{n}\right)\left[\wp\left(\gamma_{n+1}+\gamma_{n}\right)-\wp\left(\gamma_{n+1}-\gamma_{n}\right)\right]\left[\wp^{\prime}\left(\gamma_{n+1}+\gamma_{n}\right)-\wp^{\prime}\left(\gamma_{n+1}-\gamma_{n}\right)\right]^{-1}, \\
c_{n}=\left(\alpha_{1 n}-\alpha_{2 n}\right)^{-1}\left[\zeta\left(\gamma_{n+1}-\gamma_{n}\right)-\zeta\left(\gamma_{n+1}+\gamma_{n}\right)+2 \zeta\left(\gamma_{n}\right)\right] .
\end{gathered}
$$

Здесь $\gamma_{n}=\gamma_{1 n}$ и $v_{n}$ - произвольные функции, $\alpha_{1 n}$ и $\alpha_{2 n}$ определяются из соотношения:

$$
\begin{aligned}
& \alpha_{1, n+1}=-v_{n+1}+\zeta\left(\gamma_{n+1}\right)+\frac{\alpha_{1 n}}{\alpha_{1 n}-\alpha_{2 n}} \zeta\left(\gamma_{n+1}-\gamma_{n}\right)+\frac{\alpha_{2 n}}{\alpha_{1 n}-\alpha_{2 n}} \zeta\left(\gamma_{n+1}+\gamma_{n}\right), \\
& \alpha_{2, n+1}=-v_{n+1}-\zeta\left(\gamma_{n+1}\right)-\frac{\alpha_{1 n}}{\alpha_{1 n}-\alpha_{2 n}} \zeta\left(\gamma_{n+1}-\gamma_{n}\right)-\frac{\alpha_{2 n}}{\alpha_{1 n}-\alpha_{2 n}} \zeta\left(\gamma_{n+1}+\gamma_{n}\right) .
\end{aligned}
$$

Рассмотрим временну́ю динамику, где $t=t_{1}, M^{(01)}=\chi_{n}^{(0)}+\operatorname{diag}\left(v_{n}^{(0)}, v_{n+1}^{(0)}\right)$. Для матрицы Бейкера-Ахиезера $\widehat{\Psi}_{n}(t)$ имеем $\widehat{\Psi}_{n t}=M_{n} \widehat{\Psi}_{n}$, где $M_{n}=\chi_{n}+\operatorname{diag}\left(v_{n}, v_{n+1}\right)+O\left(k^{-1}\right)$. Из совместности переменных $n$ и $t$ получаем нелинейную систему для $\left(c_{n}(t), v_{n}(t)\right)$ :

$$
\begin{gathered}
\dot{c}_{n+1}=c_{n+1}\left(v_{n+1}-v_{m}\right), \quad \dot{v}_{n+1}=c_{n+2}-c_{n+1}+\varkappa_{n+1}-\varkappa_{n}, \\
\chi_{n}^{22}=k-v_{n}+\varkappa_{n} k^{-1}+O\left(k^{-2}\right) .
\end{gathered}
$$

Эта система является дискретизацией так называемого "уравнения Кричевера-Новикова" из [1]. Коэффициент $\varkappa_{n}$ можно вычислить явно, используя динамику параметров Тюрина. В следующей заметке мы рассмотрим двух (и более) точечные конструкции ранга $l-1$. Там появляется ряд новых феноменов.

\section{СПИСОК ЛИТЕРАТУРЫ}

[1] Кричевер И. М., Новиков С. П. // УМН. 1980. Т. 35. №6. С. 47-68. [2] Кричевер И. М. // Функц. анализ и прил. 1978. Т. 12. № 3. С. 20-31. 